\title{
Ausblick und mögliche Anschlussfragen
}

Gesamtüberblick: Die Ergebnisse der Entwicklung des Instruments lassen sich für zukünftige Anschlussfragestellungen weiterentwickeln. Das betrifft Möglichkeiten der Weiterentwicklung des Instruments besonders aufgrund gewisser limitierender Aspekte der zweiten quantitativen Analyse (Abschnitt 11.1). Außerdem lassen sich für das Instrument Ableitungen bezüglich des Einsatzes sowohl in Leistungs- als auch in Lernsituationen treffen (Abschnitt 11.2). Allgemeiner lassen sich weitere Anschlussfragen aus den Erkenntnissen dieser Arbeit für die didaktische Forschung und die Praxis ableiten (Abschnitt 11.3). Abschließend wird ein kurzes Fazit gezogen (Abschnitt 11.4).

\subsection{Weiterentwicklungsmöglichkeiten des Instruments}

Ein möglicher Ausblick für die weitere Forschung ist die Weiterentwicklung des dargestellten Instruments zur sprachlichen Variation von Textaufgaben im Hinblick auf zwei Aspekte. Der erste Aspekt ist die Konstruktion von Testaufgaben durch das Instrument und die Schätzung der Aufgabenschwierigkeit sowie die Feststellung des Einflusses der Faktoren auf die Aufgabenschwierigkeit in einem kontrollierten und systematisch aufgebauten mathematischen Test. Neben der Erweiterung der Erkenntnisse der bisherigen Arbeit bezüglich der Schätzung der Aufgabenschwierigkeit und des Effektes der Faktoren auf die Aufgabenschwierigkeit ist der zweite Aspekt, der für die Konstruktion eines kontrolliert und systematisch aufgebauten Tests notwendig ist, die Betrachtung von fachlichen und kontextuellen Merkmalen. Das bedeutet, dass noch unklar ist, ob die Veränderung von Aufgabentypen mit der Veränderung der sprachlichen Faktoren einhergeht. Es ist beispielsweise nicht sicher, ob ein sequenzieller Aufgabentyp, der mit

D. Bednorz, Sprachliche Variationen von mathematischen Textaufgaben, Bielefelder Schriften zur Didaktik der Mathematik 5, https://doi.org/10.1007/978-3-658-33003-3_11 
dem erklärenden Faktor assoziiert ist, fachlich und kontextuell in einen ikonischen Aufgabentyp verändert werden kann, sodass der ikonische Aufgabentyp mit dem komprimierenden Faktor assoziiert ist (bleibt). Das Ziel der Weiterentwicklung des Instruments ist entsprechend die Entwicklung von Basisaufgaben für alle fünf Aufgabentypen und die Veränderung sowie Evaluation dieser Basisaufgaben. Darüber hinaus können die Basisaufgabentypen wie erwähnt dazu dienen, ein gezieltes und systematisch aufgebautes Testdesign zu erstellen, um dadurch die Aufgabenschwierigkeiten der unterschiedlichen Faktoren, aber auch Aufgabentypen zu bestimmen. Damit könnten die Aufgabentypen anders als in der jetzigen Arbeit, in der ein vorhandener Datensatz genutzt wurde, nach Schwierigkeit skaliert werden.

\subsection{Instrument für Leistungs- und Lernsituationen}

Neben den Potenzialen des Instruments für mögliche Folgeuntersuchungen bieten die Feststellung von sprachlichen Faktoren und die Ermittlung von Aufgabentypen, die in Beziehung zu den sprachlichen Faktoren stehen, die Möglichkeit, diese für Leistungs- und Lernsituationen zu nutzen.

Leistungssituationen: Für Leistungssituationen bietet sich das dargestellte Instrument dazu an, die Veränderung der sprachlichen Faktoren zur Reduktion der Textschwierigkeit zu verwenden. Das könnte dabei helfen, den allgemeinen sprachlichen Einfluss bei Textaufgaben in mathematischen Leistungssituationen zu reduzieren, oder aber als Hilfestellung in Tests für Probandinnen und Probanden mit geringer sprachlicher Kompetenz dazu dienen, die tatsächliche mathematische Fähigkeit der Personen für einen mathematischen Test zu bestimmen.

Lernsituationen: Für Lernsituationen bietet sich das Instrument zur sprachlichen Variation von mathematischen Textaufgaben ebenfalls an. Im Hinblick auf die Bestimmung des Effektes der sprachlichen Faktoren und des Zusammenhangs zwischen den Faktoren und Aufgabentypen können sprachliche Lernprozesse durch Textaufgaben aufgebaut werden. So zeigen die Ergebnisse der Konzeptualisierung des Instruments, dass es unter dem Gesichtspunkt der Sprachförderung sinnvoll ist, sprachlich komprimierende ikonische Aufgabentypen zu nutzen. So bieten unterschiedliche Darstellungen eine Hilfe an und gleichzeitig können bedeutende Textmerkmale der Bildungs- und Fachsprache rezeptiv aufgenommen und produktiv genutzt werden. Darauf aufbauend können rechnerische Aufgabentypen, die sprachlich beschreibend, sachliche Aufgabentypen, 
die sprachlich informativ, oder fachliche Aufgabentypen, die sprachlich instruktiv sind, verwendet werden. Sequenzielle sprachlich erklärende Aufgaben haben in dem konzipierten Instrument die höchste Anforderung. Gleichzeitig macht die Auflistung deutlich, dass es durch die Verbindung von sprachlichen und fachlich-kontextuellen Merkmalen ebenfalls möglich ist, sprachliche Lernziele durch die sprachlichen Faktoren und fachliche Lernziele durch die Aufgabentypen zu formulieren und damit auch geeignete sprachliche und fachlich-didaktische Maßnahmen zu antizipieren.

\subsection{Anschlussfragen für die didaktische Forschung und Praxis}

Für das Instrument zur sprachlichen Variation stellt sich die Frage, ob dieses universell gültige sprachliche Variationen beinhaltet, also ob es auch fächerübergreifend - beispielsweise für Aufgabenstellungen in den naturwissenschaftlichen Fächern - gilt, oder ob die Faktoren nur die sprachlich-kulturelle Vielfalt sprachlicher Praktik in durch Mathematik bestimmten Texten reproduzieren. Es würde sich hierbei ein Vergleich der Faktoren von mathematischen und anderen naturwissenschaftlichen Texten anbieten, um zu prüfen, welche Gemeinsamkeiten vorhanden sind. Ebenfalls ist offen, ob die von den Lernenden produzierten Texte im Mathematikunterricht - ob in mündlicher oder schriftlicher Form - ähnliche systematische Muster des sprachlichen Gebrauchs zeigen.

Aufgrund der Verbindung der sprachlichen Grundlagen durch die Faktoren mit fachlichen Aspekten mittels der Aufgabentypen besteht eine direkte Übertragungsmöglichkeit in die Praxis. Mathematiklehrkräfte besitzen im Generellen die Expertise, trotz sprachlicher Fortbildungsmaßnahmen, mathematikdidaktische Maßnahmen zu verwenden, die eine enge Verbindung zur Mathematik besitzen. Mathematiklehrkräfte haben die Fähigkeit, geeignete Mathematikaufgaben zu wählen und diese gegebenenfalls dem Zweck anzupassen. Die Verbindung von fachlichen und sprachlichen Aspekten durch das Instrument bietet diese Möglichkeit in besonderer Weise. Mathematiklehrkräfte können spezifische Aufgabentypen sowohl aus fachlichen als auch aus sprachlichen Motiven auswählen. Diese Verbindung scheint eine besondere Stärke dieses Variationsmodells zu sein, das auf dem Registerbegriff basiert und Aspekte der Textverständlichkeit mit mathematikdidaktischen Kriterien des Lehrens und Lernens kombiniert. 


\subsection{Fazit}

Sprache hat eine hohe Relevanz für das Lehren und Lernen im Mathematikunterricht. Wenn sprachliche Voraussetzungen bei Lernenden im Mathematikunterricht fehlen, können Schwierigkeiten entstehen, mathematische Kompetenzen zu erlangen oder zu zeigen. Gerade für mathematische Textaufgaben, die in vielerlei Hinsicht eine hohe Bedeutung für das Lehren und Lernen im Mathematikunterricht haben, zeigen sich noch Möglichkeiten der Ergänzung von sprachlichen Anpassungsstrategien für Lern- und Leistungssituationen. Aus diesem Grund wurde in dieser Arbeit die Entwicklung eines Instruments zur sprachlichen Variation von Textaufgaben forciert. Das Ziel dieser Arbeit war es, durch die Entwicklung eines Instruments zur sprachlichen Variation von mathematischen Textaufgaben einen Beitrag zu der Frage zu leisten, wie Textaufgaben angepasst werden können, um das Lern- und Leistungsverhalten von Lernenden zu verbessern und adaptiv auf mögliche Hürden einzugehen. Durch die vorliegende Arbeit lassen sich Ableitungen für die Entwicklung von mathematischen Leistungstests, für die unterrichtliche Gestaltung unter der Perspektive eines sprachlich integrierten Mathematikunterrichts und für die Aus- und Fortbildung von Lehrkräften treffen.

Open Access Dieses Kapitel wird unter der Creative Commons Namensnennung 4.0 International Lizenz (http://creativecommons.org/licenses/by/4.0/deed.de) veröffentlicht, welche die Nutzung, Vervielfältigung, Bearbeitung, Verbreitung und Wiedergabe in jeglichem Medium und Format erlaubt, sofern Sie den/die ursprünglichen Autor(en) und die Quelle ordnungsgemäß nennen, einen Link zur Creative Commons Lizenz beifügen und angeben, ob Änderungen vorgenommen wurden.

Die in diesem Kapitel enthaltenen Bilder und sonstiges Drittmaterial unterliegen ebenfalls der genannten Creative Commons Lizenz, sofern sich aus der Abbildungslegende nichts anderes ergibt. Sofern das betreffende Material nicht unter der genannten Creative Commons Lizenz steht und die betreffende Handlung nicht nach gesetzlichen Vorschriften erlaubt ist, ist für die oben aufgeführten Weiterverwendungen des Materials die Einwilligung des jeweiligen Rechteinhabers einzuholen.

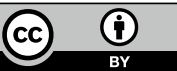

